\section{Epigenetic Modulation of BDNF Gene in Patients with Major Depressive Disorder}

To the Editor:

M ajor depressive disorder (MDD) and bipolar disorder (BD) are prevalent and disabling mood disorders, characterized by recurring mood fluctuations over the life span (1), with potential overlapping genetic risk factors $(2,3)$.

Brain-derived neurotrophic factor (BDNF), an important neurotrophin influencing synaptic plasticity, has been extensively investigated as a plausible functional candidate gene underlying the predisposition for developing mood disorders $(4,5)$, and DNA methylation represents an epigenetic mechanism contributing to its transcriptional regulation.

Recently, we observed selective alterations of BDNF gene transcription in peripheral blood mononuclear cells (PBMCs) of BDII versus $B D I$ subjects and healthy control subjects, on stable pharmacologic treatment, suggesting that DNA methylation at the gene promoter is likely to play an important role in such changes (6). In particular, we found a significant BDNF gene expression downregulation and, consistently, a hypermethylation of the BDNF promoter region in BDII patients. Among the factors potentially influencing methylation patterns, including, for instance, age, phase of illness, diet, and trauma exposure, we suggested that pharmacologic treatments might play a crucial role, in light of a significant association in our study sample between reduced methylation patterns and mood stabilizer treatment, particularly lithium and valproic acid. Although this specific association was more evident in BDI patients, mainly those treated with mood stabilizers, BDIl subjects, most of whom received a combination of mood stabilizers and antidepressants, showed hypermethylation of the BDNF promoter region.

To further investigate epigenetic mechanisms in mood disorders and to better characterize the role of BDNF, we extended our analysis to a group of patients with MDD $(n=41)$ on stable pharmacologic treatment, along with an age-matched group of healthy control subjects $(n=44)$.

The study was conducted with the appropriate ethical approval, and all subjects provided written informed consent before enrollment. Diagnoses were made by the administration of semistructured interviews based on DSM-IV criteria (Structured Clinical Interview for DSM [SCID]-I and SCID-II) $(7,8)$, and control subjects were volunteers without any psychiatric disorder, as determined by the nonpatient edition of the SCID (9). Preparation of nucleic acids, analysis of BDNF gene expression, and DNA methylation at gene promoter paralleled methods described in detail elsewhere (6).

A significant reduction of BDNF gene expression in MDD subjects $(.45 \pm .10 ; p<.05$, according to unpaired $t$ test) versus controls $(1.00 \pm .21$; Figure $1 \mathrm{~A})$ was observed. In addition, an increase of DNA methylation at BDNF gene promoter was observed in MDD patients, compared with control subjects $(32.52 \% \pm 2.03 \%$ vs. $24.04 \% \pm 2.08 \% ; p<.05$, according to unpaired $t$ test; Figure 1B). When data were stratified on the basis of pharmacotherapy, patients on antidepressant drugs alone (i.e., selective serotonin and selective norepinephrine reuptake inhibitors) showed a higher level of DNA methylation of the BDNF promoter, compared with patients receiving combination treatments of antidepressants plus mood stabilizers $(37.15 \% \pm 3.32 \%$ vs. $27.14 \% \pm 3.03 \% ; p=.042$, according to unpaired $t$ test; Figure 1C). When data were stratified according to mood state (i.e., major depressive episode; euthymia), no significant difference could be observed (Figure 1D). However, depending on mood state, data stratification, taking into account the pharmacotherapy, showed an even much higher level of DNA methylation of the BDNF promoter in depressed subjects treated with antidepressants $(42.35 \pm 4.56)$, compared with patients receiving additional mood stabilizers $(27.73 \pm 3.63 ; p=.02$, according to unpaired $t$ test). No changes were observed in euthymic patients (Figure 1E).

Taken as a whole, these findings seem to provide additional evidence in relation to BDNF gene transcription reduction in the PBMCs of MDD patients, in accordance with previous observations in BDIl patients (6) and epigenetic regulation of BDNF transcription, thus confirming its role as a potential biomarker in mood disorders. Specific differences among MDD subjects in BDNF gene regulation may depend, at least partially, on different treatment therapies, as already hypothesized for BD patients (6). Moreover, epigenetic alterations in MDD may differentially occur during specific phases of illness in patients on various pharmacologic treatments. Furthermore, our findings seem to further confirm that mood stabilizers are associated with reduced DNA methylation of the BDNF promoter in depressed subjects compared with antidepressant drugs. The presence of such associations seems to indicate BDNF regulation as a key target for such drugs. It has already been suggested that the evaluation of BDNF levels may be a general biomarker of neuronal dysfunction (particularly in BD) and that an effective treatment may reverse such alterations (10). The present findings document additional evidence for BDNF as a possible peripheral biological marker in mood disorders, further stressing the relevance of epigenetic mechanisms in the pathophysiology of major psychoses (11). Finally, data on pharmacotherapy outline the different effects of antidepressants and mood stabilizers on DNA methylation patterns in MDD, possibly depending on disease phase as well-another critical issue with relevant clinical implications (12). Further comparisons across larger samples of unipolar and bipolar subjects, including drug-free patients, in different illness phases are warranted to confirm these results.

\section{Claudio D'Addario, ${ }^{a}$ Bernardo Dell'Osso, ${ }^{b, c, *}$ \\ Daniela Galimberti,, Maria Carlotta Palazzo, b,c Beatrice Benatti, ${ }^{,}, c$ Andrea Di Francesco, ${ }^{a}$ Elio Scarpini, ${ }^{c}$ A. Carlo Altamura, ${ }^{b, c}$ Mauro Maccarrone $e^{a, d}$}

${ }^{a}$ Department of Biomedical Sciences, University of Teramo, Teramo; ${ }^{b}$ Departments of Psychiatry, University of Milan, and 'Department of Pathophysiology and Transplantation, University of Milan, Fondazione IRRCS Ca' Granda, Ospedale Maggiore Policlinico, Milan; and 'European Center for Brain Research Santa Lucia Foundation, Rome, Italy.

Authors CDA and BDO contributed equally to this work.

*Corresponding author E-mail: bernardo.dellosso@unimi.it or bernardo.dellosso@ policlinico.mi.it.

This work was supported by grants from the Monzino Foundation, Programma Strategico RF 2007, conv. PS39 (Italian Ministry of Health), TERCAS 2009-2012 and Progetti di Ricerca di Interesse Nazionale (20077R93XF_004). ADF was supported by a "Fondazione TERCAS-Progetto Speciale Assegni di Ricerca 2011-2013" fellowship.

BDO has been in the Speaker Bureau of Astra Zeneca, Bristol Myers Squibb, JanssenCilag, Eli Lilly, Pfizer, Glaxo Smith Kline, Lundbeck, Cyberonics and Italfarmaco. ACA is a consultant for Roche, Merck, Astra Zeneca, Bristol Myers Squibb, Janssen-Cilag, and Lundbeck. The other authors report no biomedical financial interests or potential conflicts of interest. 
A)

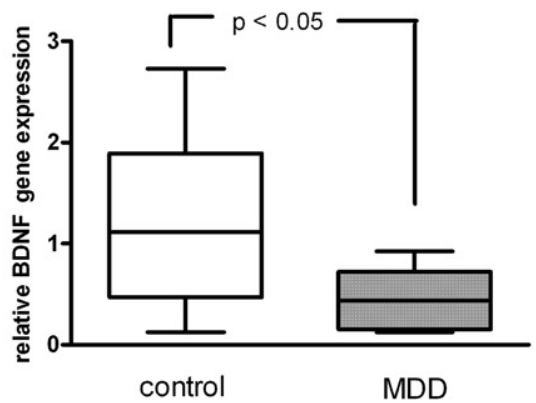

B)

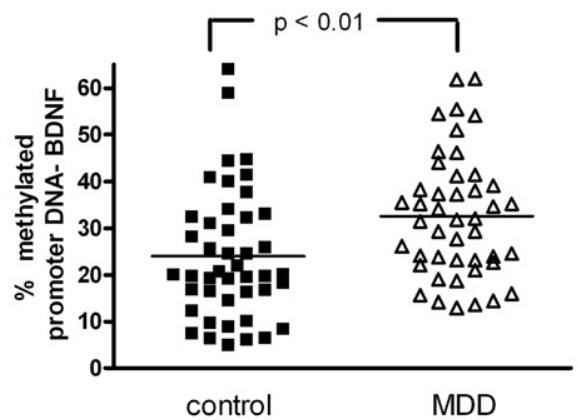

C)

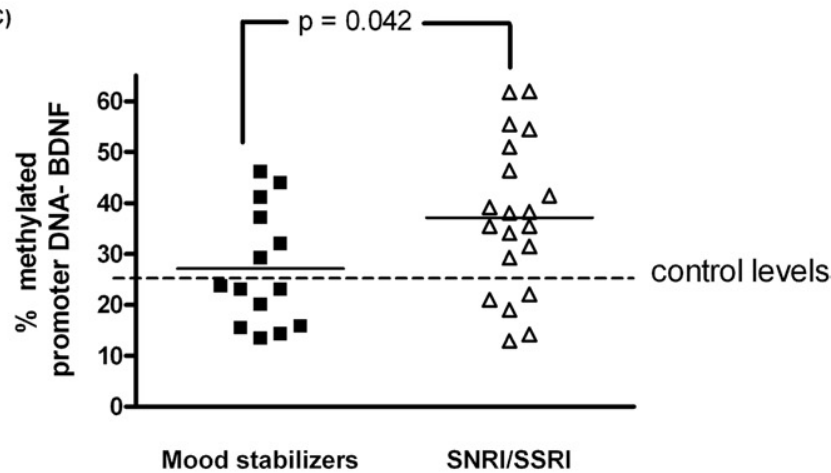

D)

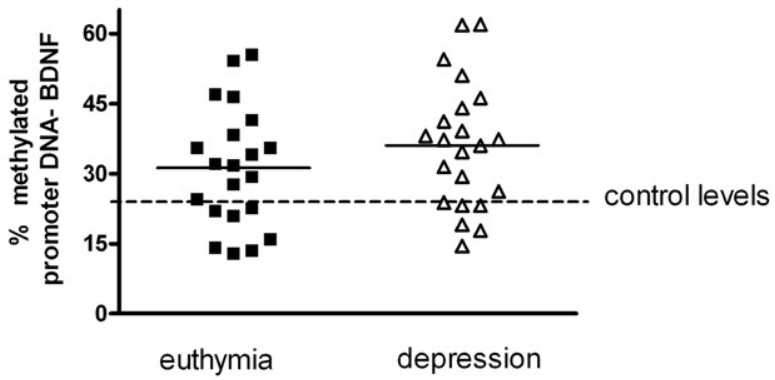

E)

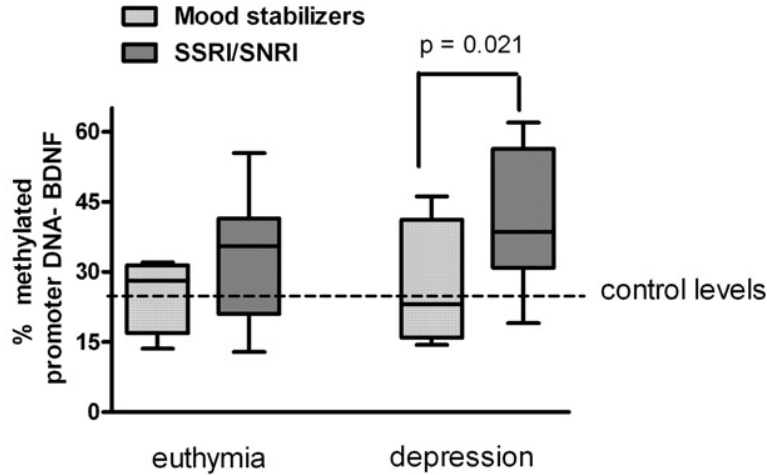

1. American Psychiatric Association (1994): Diagnostic and Statistical Manual of Mental Disorders, 4th ed. Washington, DC: American Psychiatric Press.

2. Tsuang MT, Faraone SV (1990): The Genetics of Mood Disorders. Baltimore, MD: Johns Hopkins University Press.

3. Craddock N, Jones I (2001): Molecular genetics of bipolar disorder. Br J Psychiatry 178:128-133.

4. Duman RS, Malberg J, Nakagawa S, D'Sa C (2000): Neuronal plasticity and survival in mood disorders. Biol Psychiatry 48:732-739.

5. Nakata K, Ujike H, Sakai A, Uchida N, Nomura A, Imamura T, http:// www.ncbi.nlm.nih.gov/pubmed?term =Katsu\%20T\%5BAuthor\%5D\& cauthor $=$ true\&cauthor_uid=12524161 et al. (2003): Association study of the brain-derived neurotrophic factor (BDNF) gene with bipolar disorder. Neurosci Lett 337:17-20.

6. D'Addario C, Dell'Osso B, Palazzo MC, Benatti B, Lietti L, Cattaneo E, et al. (2012): Selective DNA methylation of BDNF promoter in bipolar disorder: Differences among patients with BDI and BDII. Neuropsychopharmacology 37:1647-1655.

7. First MB, Spitzer RL, Gibbon L, Williams JBV (2002): Structured Clinical Interview for DSM-IV-TR Axis I Disorders, Research Version, Patient Edition (SCID-I/P). New York: New York State Psychiatric Institute, Biometric Research.

8. First MB, Gibbon M, Spitzer RL, Williams, JBW, Benjamin LS (1997): Structured Clinical Interview for DSM-IV Axis II Personality Disorders (SCID-II). Washington, DC: American Psychiatric Press

9. First MB, Spitzer RL, Gibbon L, Williams JBV (2002): Structured Clinical Interview for DSM-IV-TR Axis I Disorders, Research Version, Non-Patient Edition (SCID-I/NP). New York: New York State Psychiatric Institute, Biometric Research.

10. Grande I, Fries GR, Kunz M, Kapczinski F (2010): The role of BDNF as a mediator of neuroplasticity in bipolar disorder. Psychiatry Investig 7:243-250.

11. Mill J, Petronis A (2009): The relevance of epigenetics to major psychosis. In: Ferguson-Smith AC, Greally JM, Martienssen RA, editors. Epigenomics (Part IV). New York: Springer, 411-434.

12. Uher R (2008): The implications of gene-environment interactions in depression: Will cause inform cure? Mol Psychiatry 13:1070-1078.

http://dx.doi.org/10.1016/j.biopsych.2012.07.009
Figure 1. Levels of brain-derived neurotrophic factor (BDNF) messenger (m)RNA in peripheral blood mononuclear cells (PBMCs) from patients diagnosed with major depressive disorder (MDD). Box plots with whiskers from minimum to maximum represent $2^{-D D C t}$ values calculated by the Delta-Delta Ct (DDCt) method. (A) Means of mRNA levels are expressed relative to control subjects. (B) Amount of methylated DNA in the promoter region of BDNF in PBMCs from both control and MDD subjects and from MDD patients alone (C) according to different therapies (mood stabilizers or antidepressant drugs), (D) mood state, or (E) both. Scatter plots with mean values are shown. SNRI, serotonin-norepinephrine reuptake inhibitor; SSRI, selective serotonin reuptake inhibitor. 